Nephrologe 2011 - 6:469-469

DOI 10.1007/s11560-011-0549-z

(c) Springer-Verlag 2011

\author{
H. Haller ${ }^{1} \cdot$ J. Hoyer $^{2}$ \\ ${ }^{1}$ Klinik für Nieren- und Hochdruckerkrankungen, Medizinische Hochschule Hannover \\ 2 Klinik für Innere Medizin und Nephrologie, \\ Universitätsklinikum der Philipps-Universität Marburg
}

\title{
Nieren und Hypertonie
}

tion bei der Reduktion des kardiovaskulären Risikos und hypertensiver Endorganschäden vergleicht Hoyer diese Studien mit dem ansonsten gut belegten Wissensstand und gibt Hinweise zur Umsetzung einer nichtmedikamentösen Blutdruckbehandlung mittels diätetischer Kochsalzreduktion.

Bei Kindern und Jugendlichen wird eine Niereninsuffizienz häufig durch eine schwere Hypertonie kompliziert und kann zu erheblichen therapeutischen Problemen führen. Auf Basis ihrer exzellenten Hypertoniestudie (ESCAPE) beschreibt E. Wühl in ihrem Beitrag die Besonderheiten der antihypertensiven Therapie im Kindes- und Jugendalter und weist auf spezifische Aspekte der renalen Protektion hin.

In den letzten Jahren hat es eine Reihe von medikamentösen Neuentwicklungen gegeben, die nicht nur auf die Hemmung des RAAS abzielen. Kreutz und Kintscher stellen in ihrem Artikel die relevanten Neuentwicklungen antihypertensiver Substanzen vor und bewerten deren Chancen zur Etablierung als klinisch einsetzbare Antihypertensiva. Darüberhinaus geben Hoyer und Haller in einem Update-Artikel einen Überblick über neue Entwicklungen und Studien aus der experimentellen und klinischen Hypertensiologie. Die Hypertensiologie ist ein dynamisches Fachgebiet, welches sich permanent auf Ebene der präventiven Medizin, bei der Entwicklung neuartiger interventioneller Therapieformen und bei der der Etablierung neuer Pharmaka weiterentwickelt - wir hoffen, dass Sie durch die Beiträge dieses Hefts gute Hinweise für die tägliche Praxis mit Hypertoniepatienten erhalten.
Mit kollegialen Grüßen

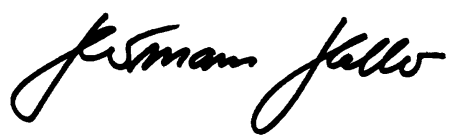

H. Haller

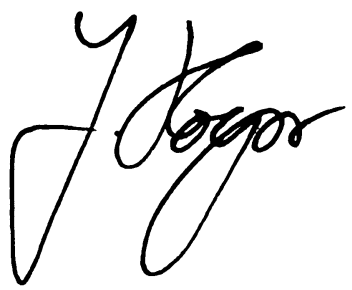

J. Hoyer

\section{Korrespondenzadresse \\ Prof. Dr. H. Haller}

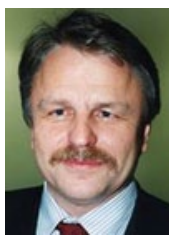

Klinik für Nieren- und Hochdruckerkrankungen, Medizinische Hochschule Hannover Carl-Neuberg-Str. 1, 30625 Hannover Haller.Hermann@ mh-hannover.de

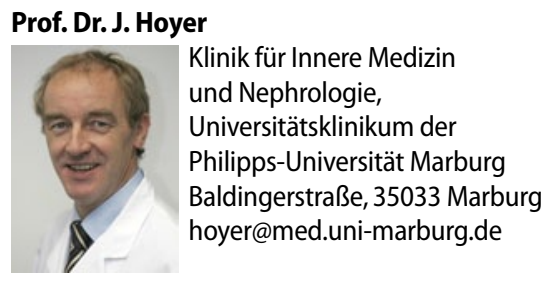

